\title{
Iodine prophylaxis, goitre and thyroid autoimmunity in Sri Lanka CN Wijeyaratne ${ }^{1}$, A Jayasinghe ${ }^{2}$, DGH de Silva ${ }^{3}$, AB Parkes ${ }^{4}$, JH Lazarus ${ }^{4}$ and LDKE Premawardhana ${ }^{4}$
}

\section{Introduction}

Iodine deficiency is a major public health problem. Over one third of the world's population lived in iodine deficient areas in 1998 [1]. Although goitre is the first and most obvious consequence, lack of iodine may give rise to a spectrum of disorders (iodine deficiency disorders, IDD). The developing brain is the most vulnerable target. Mild neuro-intellectual impairment (often unrecognised unless carefully tested for) and severe mental and neurological damage as seen in cretinism are the two ends of the spectrum [2]. A meta-analysis of 18 studies of intellectual performance of iodine deficient children in comparison to iodine sufficient peers revealed a reduced IQ of 13.5 points [3]. These effects on neuro-intellectual development and the adverse effects of iodine deficiency on neonatal mortality and pregnancy outcome are the main reasons for promoting urgent iodine prophylaxis. The WHO and UNICEF highlighted the need for iodine prophylaxis in their own spheres of influence, but the International Council for Control of Iodine Deficiency Disorders (ICCIDD) was set up (in 1985) for the sole purpose of promoting worldwide sustainable iodine nutrition. Salt iodination is the currently recommended strategy for iodine provision. In 1998, there were 98 countries (out of 191 with iodine deficiency) with legislation for salt iodination [1].

Government legislation in 1993 made salt iodination compulsory in Sri Lanka. Several studies published previously, reported a high prevalence of goitre in many parts of Sri Lanka [4-6]. Goitre size in these studies was assessed by palpation using WHO criteria. Although no studies on iodine consumption or excretion had been done, the assumption that iodine deficiency was the proximate cause for high goitre prevalence was a valid one. One study established low iodine concentrations in drinking water.

The most recent and largest epidemiological study of goitre prevalence was published in 1989 [7]; nearly 60000 schoolgirls between 11 and 17 years of age were studied in several areas in Sri Lanka. Goitre prevalence was established by teams of technicians who visited schools in a number of localities. Some areas had a high goitre prevalence (surprisingly including some coastal areas), whereas more urban areas such as Colombo had low goitre prevalence [8].

\begin{abstract}
Although salt iodination is an effective method of iodine prophylaxis, supervision of the iodination process and monitoring of the iodine content of commercially available salts are vital in ensuring adequate and sustained iodine delivery to the consumer. There is evidence to suggest that the iodine content of salt obtained from several commercial outlets in the country is inappropriate; $52 \%$ of the commercially available salt preparations were reported to contain excess concentrations of iodine, and only $32 \%$ contained the recommended concentration of 20-40 parts per million [9]. Both low and high salt iodine concentrations may be harmful. The large proportions of commercial salts with iodine content more than that recommended has the potential to increase the risk of iodine induced complications.
\end{abstract}

\section{Has salt iodination been effective in Sri Lanka?}

The beneficial effects of iodine prophylaxis may take time to manifest. There is evidence from some areas where iodine prophylaxis has been undertaken, that goitre prevalence (or thyroid volume, where measured) decreased within a year, whereas in other areas a longer time was needed [10, 11].

We undertook two studies (in 1998 and 2001) to assess ultrasound measured thyroid volume (TV) in schoolgirls aged between 11 and 17 years from areas with previous low, intermediate and high goitre prevalence $[8,12]$ (Tables 1and 2). TV was compared to normative data from the corrected WHO/ICCIDD dataset for schoolgirls from iodine replete areas [13]. There was a significant reduction in TV and goitre prevalence in 2001 compared to

Table 1. Anthropometric data and thyroid volume of 11-17 year old schoolgirls in 1998 and 2001

\begin{tabular}{lrrr}
\hline & 1998 & 2001 & $p$ \\
\hline Body surface area $\left(\mathrm{m}^{2}\right)$ & $1.26 \pm 0.1$ & $1.36 \pm 0.1$ & $<0.001$ \\
Lean body mass $(\%)$ & $28.3 \pm 4.9$ & $32.4 \pm 7.7$ & $<0.001$ \\
Age (years) & $13.7 \pm 1.3$ & $15 \pm 1.2$ & $<0.001$ \\
Thyroid volume $(\mathrm{mL})$ & $7.7 \pm 3.7$ & $5.8 \pm 2.2$ & $<0.001$ \\
\hline
\end{tabular}

Data are expressed as mean \pm SD

${ }^{1}$ Department of Obstetrics and Gynaecology, Faculty of Medicine, Kynsey Road, Colombo, Sri Lanka; ${ }^{2}$ Department of Community Medicine, Faculty of Medicine, University of Peradeniya, Sri Lanka; ${ }^{3}$ Department of Paediatrics, Faculty of Medicine, University of Kelaniya, Sri Lanka; ${ }^{4}$ Section of Endocrinology, Department of Medicine, University of Wales College of Medicine, Cardiff, UK.

Correspondence: CNW, e-mail: <mandika59@hotmail.com> (Competing interests: none declared). Received 10 September 2004 and accepted 29 November 2004. 
1998 (related both to age and to body surface area). These data were further corroborated by a Sri Lanka Medical Research Institute (MRI)/UNICEF survey done recently using palpation as a method of goitre estimation [14]. This study found a goitre prevalence of 16.3-26.2 \% with decreased goitre rates in most provinces. We also demonstrated median urine iodine (UI) concentrations in the iodine replete range in schoolgirls from the areas we studied. There was a significant reduction in goitre prevalence compared to age and body surface area between 1998 and 2001, while mean UI concentration was maintained in the iodine replete range and was similar in the two studies (Table 2). The MRI/UNICEF survey too found adequate median UI concentrations in the large majority of the areas surveyed (except in the Uva province) [14]. UI is a good surrogate marker of iodine intake as a large proportion of ingested iodine is excreted in urine.

Table 2. Goitre prevalence and urine iodine concentration in schoolgirls in 1998 and 2001

\begin{tabular}{lcc}
\hline Goitre prevalence & 1998 & 2001 \\
\hline Age related $(\%)$ & 20.2 & 2.9 \\
Body surface area related $(\%)$ & 40.8 & 11.6 \\
Urine iodine $(\mu \mathrm{g} / \mathrm{L})$ & $149.5 \pm 78.4$ & $158.9 \pm 9.5^{*}$ \\
\hline
\end{tabular}

* p-0.34.

The significant reduction in TV and goitre prevalence, and UI concentrations in the iodine replete range indicate a positive impact of the salt iodination programme, which needs to be encouraged and developed.

\section{lodine and thyroid autoimmunity in Sri Lanka}

We also examined thyroid autoimmune markers in the schoolgirls we studied in the two projects. There was a significant reduction in the prevalence of thyroid antibodies in schoolgirls aged $11-17$ years by 2001 , and modulation in their thyroid antibody profile (Table 3 ).

\section{Table 3. Prevalence of TgAb and TPOAb in schoolgirls in 1998 and 2001}

\begin{tabular}{lrr}
\hline $\begin{array}{l}\text { Prevalence of thyroid } \\
\text { antibodies }\end{array}$ & 1998 & 2001 \\
\hline Total prevalence (\%) & 49.9 & 23.4 \\
TgAb only (\%) & 40.9 & 8.2 \\
TPOAb ( $(\mathrm{TgAb})(\%)$ & 9.0 & 15.2 \\
\hline
\end{tabular}

TgAb-anti-thyroglobulin antibodies, TPOAb-anti-thyroid peroxidase antibodies

The prevalence of anti-thyroid peroxidase antibodies (TPOAb) was less than $10 \%$ in all age groups. However, a higher prevalence of TPOAb was seen either alone $(18.2 \%)$ or in combination with anti-thyroglobulin antibodies (TgAb) $(46.9 \%$; $<<0.001)$. TPOAb, which is a more specific marker than $\mathrm{TgAb}$ of thyroid autoimmunity, was associated with subclinical thyroid disease in 2001. Is there an explanation for this evolving thyroid autoimmunity?

Thyroglobulin $(\mathrm{Tg})$, a large protein molecule, is the template for thyroid hormone production. Iodination of tyrosine residues on the $\mathrm{Tg}$ molecule under normal conditions leads to thyroxine (T4) and tri-iodothyronine (T3) synthesis. However, there is good in vitro evidence to suggest that an excess of iodine makes Tg highly immunogenic by enhancing the processing and presentation of cryptic peptide epitopes [15-17]. Whether this is a truly pathogenetic mechanism precipitating autoimmune thyroiditis or a "bystander" phenomenon is as yet undecided. The varying content of iodine in commercially available salts (with an excess of iodine in $52 \%$ of salt samples analysed) may be relevant in this regard $[5,12]$. This acute response to excess iodine seems to wane over a period, as demonstrated by a fall in the prevalence of $\operatorname{TgAb}$ between 1998 and 2001, but the emergence of TPOAb and its association with subclinical thyroid disease is a worrying phenomenon. The effects on the immunogenicity of TPO and other immune enhancing effects of excess iodine need to be further elucidated.

We further investigated the high prevalence of $\mathrm{TgAb}$ in schoolgirls in 1998 and its pathogenetic significance by analysing epitope recognition patterns of these antibodies [18]. Human monoclonal antibodies raised against epitope clusters on the TgAb molecule were used to characterise $\mathrm{TgAb}$ from Sri Lankan schoolgirls. In vitro studies have shown a characteristic restricted epitope recognition pattern in $\mathrm{TgAb}$ obtained from people with autoimmune thyroid disease (AITD). People without AITD showed an unrestricted pattern $[19,20]$. The TgAb obtained from our schoolgirls demonstrated an unrestricted epitope recognition pattern, indicating in all probability a non-pathogenetic role.

What are we to make of these immune phenomena? There are reports of similar thyroid related immune changes from India [21], Germany [22] and Greece [23] where recent iodine replenishment has occurred. These studies were undertaken several years after the introduction of iodine and showed a TPOAb predominance. It is probable that these investigators missed an early $\mathrm{TgAb}$ peak. The concept of evolving thyroid autoimmunity with continuous iodine intake (early $\mathrm{TgAb}$ predominance followed by a late TPOAb predominance and associated AITD), thereby heralding a more aggressive phase of autoimmunity is worrying, and needs further clarification [24]. Urgent studies are required to examine autoimmune and biochemical thyroid dysfunction in groups particularly at risk of AITD - pregnant women and the elderly, for example.

\section{lodine prophylaxis and the future}

The control of iodine deficiency and its eventual elimination are triumphs of preventive medicine. The benefits of iodine prophylaxis far outweigh actual and theoretical disadvantages [25]. The progress made in Sri 
Lanka in preventing iodine deficiency should be commended. Nevertheless, a few cautionary points should be remembered to maximise the benefits of iodine supplementation.

1. The national programme of iodine prophylaxis should be accompanied by periodic surveys of UI as a marker of population iodine status. A dedicated laboratory should be available locally for the measurement of UI, appropriately equipped and staffed, with adequate mechanisms for quality control. Surveys in Australia [26] and USA [27] have demonstrated a fall in UI over a period of time. Clear strategies need to be in place to avoid resurgence of iodine deficiency in populations where salt consumption has fallen.

2. Adequate supervision of salt iodination should be complemented by analysis of iodine in commercially available salt samples. Wide variation in iodine concentrations and a high proportion of commercial salt samples with an excess iodine is worrying in view of possible iodine induced immune phenomena. Effective legislation and monitoring of salt iodination is essential both at the processing factory and sales points.

3. The induction of thyroid autoimmunity and a gradual modulation of thyroid autoantibodies with continuing ingestion of iodine is a newly recognised phenomenon. The change from non-pathogenic $\mathrm{TgAb}$ to potentially pathogenic TPOAb and its association with subclinical thyroid dysfunction is a cause for concern. Further large scale studies are necessary to examine the effects of such changes on groups at maximum risk, i.e. the pregnant and the elderly.

In conclusion, we would like to emphasise the short term success of the National Sri Lankan Salt Iodination Programme in reducing TV and goitre prevalence, while maintaining UI within iodine replete levels. It is encouraging that iodine induced thyrotoxicosis was not found in our sample of schoolchildren. We would like to sound a note of caution about the absence of an effective monitoring programme, as some effects of excess iodine are already apparent. The immune modulatory phenomena we have described may herald widespread AITD in the future.

\section{Acknowledgements}

We thank our collaborators Dr. PPA Smyth (Director, Endocrine Laboratory, University College Dublin, Ireland) and Dr. H Adams (Department of Radiology, Llandough Hospital, UK) for field work and ultrasound scans; Dr. G Mazziotti, Dr. OE Okosieme and Mrs Lynn Taylor (Endocrine Immunology Laboratory, Cardiff) for laboratory and data analysis; the Education Ministry and Principals of the schools where we were made to feel very welcome; all research assistants for their untiring effort, and the World Bank who funded this research through the Sri Lanka Ministry of Health. Finally we thank the schoolgirls who took part in this project, without whom these studies would have been impossible.

\section{References}

1. WHO, UNICEF and ICCIDD. Progress towards the elimination of iodine deficiency disorders (IDD). 1999, WHO, Geneva.

2. Dunn JT, Delange F. Damaged reproduction: the most important consequence of iodine deficiency. Journal of Clinical Endocrinology and Metabolism 2001; 86: 2360-3

3. Bleichrodt N, Born MP. A meta-analysis of research on iodine and its relationship to cognitive development. In: Stanbury JB, ed. The damaged brain of iodine deficiency. New York Cognizant Communication; 1994: 195-200.

4. Mahadeva K, Senthe Shanmuganathan S. The problem of goitre in Ceylon. British Journal of Nutrition 1967; 21: $341-52$

5. Deo HG, Subramaniam TAV. Iodine metabolism in children and women with endemic goitre in Ceylon. British Journal of Nutrition 1971; 25: 97-105

6. Mahadeva K, Seneviratne DA, Jayathilleke DB, Shanmuganathan SS, Premachandra P, et al. Further studies on the problem of goitre in Ceylon. British Journal of Nutrition 1968; 22: 527-34

7. Fernando MA, Balasuriya S, Herath KB, Katugampola S. Endemic goitre in Sri Lanka. Asia Pacific Journal of Public Health 1989; 3: 11-8

8. Premawardhana LDKE, Parkes AB, Smyth PPA, Wijeyaratne CN, Jayasinghe A, et al. Increased prevalence of thyroglobulin antibodies in Sri Lankan schoolchildren - is iodine the cause? European Journal of Endocrinology 2000; 143: $185-8$

9. Kumarasiri JP, Fernandopulle BMR, Lankathilake MALKN. Iodine content in commercially available iodised salt in the Sri Lankan market. Ceylon Medical Journal 1998; 43: 84-7

10. Jooste PL, Weight MJ, Lombard CJ. Short-term effectiveness of mandatory iodization of table salt, at an elevated iodine concentration, on the iodine and goitre status of schoolchildren with endemic goitre. American Journal of Clinical Nutrition 2000; 71: 75-80

11. Zimmermann MB, Hess SY, Abdou P, Toresanni T, Wegmuller R, Hurrell RF. Thyroid size and goitre prevalence after introduction of iodized salt: a 5-y prospective study in schoolchildren in Côte d'Ivoire. American Journal of Clinical Nutrition 2003; 77: 663-7

12. Mazziotti G, Premawardhana LDKE, Parkes AB, Adams $\mathrm{H}$, Smyth PPA, et al. Evolution of thyroid autoimmunity during iodine prophylaxis - the Sri Lankan experience. European Journal of Endocrinology 2003; 149: 103-10

13. Zimmermann MB, Molinari L, Spehl M, Weidinger Toth J, Poboda J, Hess S, Delange F. Toward a consensus on reference values for thyroid volume in iodine-replete schoolchildren: results of a workshop on inter-observer and inter-equipment variation in sonographic measurement 
of thyroid volume. European Journal of Endocrinology 2001; 144: 213-20

14. Medical Research Institute. Report on Prevalence of goitre and urine iodine excretion in Sri Lanka, Ministry of Health, 2001.

15. Rose NR, Saboori AM, Rasooly L, Burek CL. The role of iodine in autoimmune thyroiditis. Critical Reviews in Immunology 1997; 17:511-7

16. Rasooly L, Burek CL, Rose NR. Iodine- induced autoimmune thyroiditis in NOD-H-2 ${ }^{\mathrm{h} 4}$ mice. Clinical Immunology and Immunopathology 1996; 81: 287-92

17. Sundick RS, Bagchi N, Brown TR. The role of iodine in thyroid autoimmunity: from chickens to humans-a review. Autoimmunity 1992; 13: 61-8

18. Okosieme OE, Premawardhana LDKE, Jayasinghe A, de Silva DGH, Smyth PPA, et al. Thyroglobulin epitope recognition in a post-iodine supplemented Sri Lankan population. Clinical Endocrinology 2003; 59:190-7

19. Caturegli P, Mariotti S, Kuppers C, Burek CL, Pinchera A, Rose NR. Epitopes on thyroglobulin: a study of patients with thyroid disease. Autoimmunity 1994; 18: 41-9.

20. Ruf J, Carayon P, Lissitzky S. Various expressions of a unique anti-human thyroglobulin antibody repertoire in a normal state and autoimmune disease. European Journal of Immunology 1985; 15: 268-72

21. Marwaha RK, Tandon N, Karak AK, Gupta N, Verma K, et al. Hashimoto's thyroiditis: countrywide screening of goitrous healthy young girls in postiodization phase in
India. Journal of Clinical Endocrinology and Metabolism 2000; 85: 3798-802

22. Kabelitz M, Liesenkötter KP, Stach B, Willgerodt H, Stäblein $\mathrm{W}$, et al. The prevalence of anti- thyroid peroxidase antibodies and autoimmune thyroiditis in children and adolescents in an iodine replete area. European Journal of Endocrinology 2003; 148: 301-7

23. Zois C, Stavrou I, Kalogera C, Svarna E, Dimoliatis I, et al. High prevalence of autoimmune thyroiditis in schoolchildren after elimination of iodine deficiency in Northwestern Greece. Thyroid 2003; 13: 485-9

24. Premawardhana LDKE, Parkes AB, Mazziotti G, Lazarus JH. Autoimmune thyroiditis after elimination of iodine deficiency in Sri Lanka. Thyroid 2003; 13: 1187

25. Dunn JT. Editorial: What's happening to our iodine. Journal of Clinical Endocrinology and Metabolism 1998; 83: $3398-400$

26. Guttikonda K, Burgess JR, Hynes K, Boyages S, Byth K, Parameswaran V. Recurrent iodine deficiency in Tasmania, Australia: a salutary lesson in sustainable iodine prophylaxis and its monitoring. Journal of Clinical Endocrinology and Metabolism 2002; 87: 2809-15

27. Hollowell JG, Staehling NW, Hannon WH, Flanders DW, Gunter EW, et al. Iodine nutrition in the United States. Trends and Public health implications: iodine excretion data from National Health and Nutrition Examination Surveys I and III (1971-1974 and 1988-1994). Journal of Clinical Endocrinology and Metabolism 1998; 83: 3401-8 